\title{
Statistical Estimation for CAPM with Long-Memory Dependence
}

\author{
Tomoyuki Amano, ${ }^{1}$ Tsuyoshi Kato, ${ }^{2}$ and Masanobu Taniguchi ${ }^{2}$ \\ ${ }^{1}$ Faculty of Economics, Wakayama University, Wakayama 6408510, Japan \\ 2 Department of Applied Mathematics, School of Fundamental Science and Engineering, \\ Waseda University, Tokyo 1698555, Japan \\ Correspondence should be addressed to Tomoyuki Amano, tomchami@center.wakayama-u.ac.jp
}

Received 24 June 2011; Revised 27 August 2011; Accepted 10 September 2011

Academic Editor: Junichi Hirukawa

Copyright (C) 2012 Tomoyuki Amano et al. This is an open access article distributed under the Creative Commons Attribution License, which permits unrestricted use, distribution, and reproduction in any medium, provided the original work is properly cited.

We investigate the Capital Asser Pricing Model (CAPM) with time dimension. By using time series analysis, we discuss the estimation of CAPM when market portfolio and the error process are long-memory process and correlated with each other. We give a sufficient condition for the return of assets in the CAPM to be short memory. In this setting, we propose a two-stage least squares estimator for the regression coefficient and derive the asymptotic distribution. Some numerical studies are given. They show an interesting feature of this model.

\section{Introduction}

The CAPM is one of the typical models of risk asset's price on equilibrium market and has been used for pricing individual stocks and portfolios. At first, Markowitz [1] did the groundwork of this model. In his research, he cast the investor's portfolio selection problem in terms of expected return and variance. Sharpe [2] and Lintner [3] developed Markowitz's idea for economical implication. Black [4] derived a more general version of the CAPM. In their version, the CAPM is constructed based on the excess of the return of the asset over zero-beta return $E\left[R_{i}\right]=E\left[R_{0 m}\right]+\beta_{i m}\left(E\left[R_{m}\right]-E\left[R_{0 m}\right]\right)$, where $R_{i}$ and $R_{m}$ are the return of the $i$ th asset and the market portfolio and $R_{0 m}$ is the return of zero-beta portfolio of the market portfolio. Campbell et al. [5] discussed the estimation of CAPM, but in their work they did not discuss the time dimension. However, in the econometric analysis, it is necessary to investigate this model with the time dimension; that is, the model is represented as $R_{i, t}=\alpha_{i m}+\beta_{i m} R_{m, t}+\epsilon_{i, t}$. Recently from the empirical analysis, it is known that the return of 
asset follows a short-memory process. But Granger [6] showed that the aggregation of shortmemory processes yields long-memory dependence, and it is known that the return of the market portfolio follows a long-memory process. From this point of view, first, we show that the return of the market portfolio and the error process $\epsilon_{t}$ are long-memory dependent and correlated with each other.

For the regression model, the most fundamental estimator is the ordinary least squares estimator. However, the dependence of the error process with the explanatory process makes this estimator to be inconsistent. To overcome this difficulty, the instrumental variable method is proposed by use of the instrumental variables which are uncorrelated with the error process and correlated with the explanatory variable. This method was first used by Wright [7], and many researchers developed this method (see Reiersøl [8], Geary [9], etc.). Comprehensive reviews are seen in White [10]. However, the instrumental variable method has been discussed in the case where the error process does not follow long-memory process, and this makes the estimation difficult.

For the analysis of long-memory process, Robinson and Hidalgo [11] considered a stochastic regression model defined by $y_{t}=\alpha+\boldsymbol{\beta}^{\prime} \mathbf{x}_{t}+u_{t}$, where $\alpha, \beta=\left(\beta_{1}, \ldots, \beta_{K}\right)^{\prime}$ are unknown parameters and the $K$-vector processes $\left\{\mathbf{x}_{t}\right\}$ and $\left\{u_{t}\right\}$ are long-memory dependent with $E\left(\mathbf{x}_{t}\right)=\mathbf{0}, E\left(u_{t}\right)=0$. Furthermore, in Choy and Taniguchi [12], they consider the stochastic regression model $y_{t}=\beta x_{t}+u_{t}$, where $\left\{x_{t}\right\}$ and $\left\{u_{t}\right\}$ are stationary process with $E\left(x_{t}\right)=\mu \neq 0$, and Choy and Taniguchi [12] introduced a ratio estimator, the least squares estimator, and the best linear unbiased estimator for $\beta$. However, Robinson and Hidalgo [11] and Choy and Taniguchi [12] assume that the explanatory process $\left\{x_{t}\right\}$ and the error process $\left\{u_{t}\right\}$ are independent.

In this paper, by the using of instrumental variable method we propose the two-stage least squares (2SLS) estimator for the CAPM in which the returns of the individual asset and error process are long-memory dependent and mutually correlated with each other. Then we prove its consistency and CLT under some conditions. Also, some numerical studies are provided.

This paper is organized as follows. Section 2 gives our definition of the CAPM, and we give a sufficient condition that return of assets as short dependence is generated by the returns of market portfolio and error process which are long-memory dependent and mutually correlated each other. In Section 3 we propose 2SLS estimator for this model and show its consistency and asymptotic normality. Section 4 provides some numerical studies which show interesting features of our estimator. The proof of theorem is relegated to Section 5.

\section{CAPM (Capital Asset Pricing Model)}

For Sharpe and Lintner version of the CAPM (see Sharpe [2] and Lintner [3]), the expected return of asset $i$ is given by

$$
E\left[R_{i}\right]=R_{f}+\beta_{i m}\left(E\left[R_{m}-R_{f}\right]\right)
$$

where

$$
\beta_{i m}=\frac{\operatorname{Cov}\left[R_{i}, R_{m}\right]}{V\left[R_{m}\right]}
$$


$R_{m}$ is the return of the market portfolio, and $R_{f}$ is the return of the risk-free asset. Another Sharpe-Lintner's CAPM (see Sharpe [2] and Lintner [3]) is defined for $Z_{i} \equiv R_{i}-R_{f}$,

$$
E\left[Z_{i}\right]=\beta_{i m} E\left[Z_{m}\right]
$$

where

$$
\beta_{i m}=\frac{\operatorname{Cov}\left[Z_{i}, Z_{m}\right]}{V\left[Z_{m}\right]}
$$

and $Z_{m}=R_{m}-R_{f}$.

Black [4] derived a more general version of CAPM, which is written as

$$
E\left[R_{i}\right]=\alpha_{i m}+\beta_{i m} E\left[R_{m}\right],
$$

where $\alpha_{i m}=E\left[R_{0 m}\right]\left(1-\beta_{i m}\right)$ and $R_{0 m}$ is the return on the zero-beta portfolio.

Since CAPM is single-period model, (2.1) and (2.5) do not have a time dimension. However, for econometric analysis of the model, it is necessary to add assumptions concerning the time dimension. Hence, it is natural to consider the model:

$$
Y_{i, t}=\alpha_{i}+\beta_{i} Z_{t}+\epsilon_{i, t}
$$

where $i$ denotes the asset, $t$ denotes the period, and $Y_{i, t}$ and $Z_{t}, i=1, \ldots, n$ and $t=1, \ldots, T$, are, respectively, the returns of the asset $i$ and the market portfolio at $t$. known.

Empirical features of the realized returns for assets and market portfolios are well

We plot the autocorrelation function $(\operatorname{ACF}(l)(l$ : time lag)) of returns of IBM stock and S\&P500 (squared transformed) in Figures 1 and 2, respectively.

From Figures 1 and 2, we observe that the return of stock (i.e., IBM) shows the shortmemory dependence and that a market index (i.e., S\&P500) shows the long-memory dependence.

Suppose that an $n$-dimensional process $\left\{\mathbf{Y}_{t}=\left(Y_{1, t}, \ldots, Y_{n, t}\right)^{\prime}\right\}$ is generated by

$$
\mathbf{Y}_{t}=\boldsymbol{\alpha}+\mathbf{B}^{\prime} \mathbf{Z}_{t}+\boldsymbol{\epsilon}_{t} \quad(t=1,2, \ldots, T),
$$

where $\boldsymbol{\alpha}=\left(\alpha_{1}, \ldots, \alpha_{n}\right)^{\prime}$ and $\mathbf{B}=\left\{\beta_{i j} ; i=1, \ldots, p, j=1, \ldots, n\right\}$ are unknown vector and matrix; respectively, $\left\{\mathbf{Z}_{t}=\left(Z_{1, t}, \ldots, Z_{p, t}\right)^{\prime}\right\}$ is an explanatory stochastic regressor process, and $\left\{\boldsymbol{\epsilon}_{t}=\left(\epsilon_{1, t}, \ldots, \epsilon_{n, t}\right)^{\prime}\right\}$ is a sequence of disturbance process. The $i$ th component is written as

$$
Y_{i, t}=\alpha_{i}+\boldsymbol{\beta}_{i}^{\prime} \mathbf{Z}_{t}+\epsilon_{i, t}
$$

where $\boldsymbol{\beta}_{i}^{\prime}=\left(\beta_{i, 1}, \ldots, \beta_{i, p}\right)$.

In the CAPM, $\mathbf{Y}_{t}$ is the return of assets and $\mathbf{Z}_{t}$ is the return of the market portfolios. As we saw, empirical studies suggest that $\left\{\mathbf{Y}_{t}\right\}$ is short-memory dependent and that $\left\{\mathbf{Z}_{t}\right\}$ is long-memory dependent. On this ground, we investigate the conditions that the CAPM (2.7) 


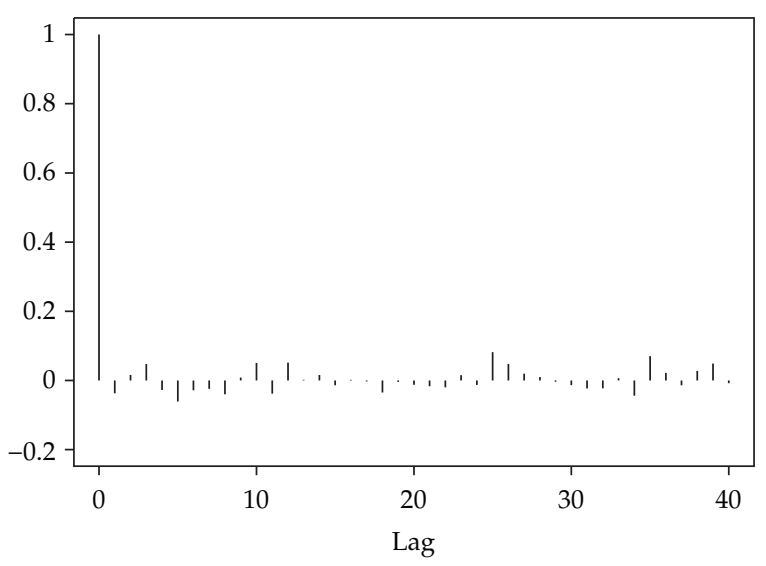

Figure 1: ACF of return of the IBM stock.

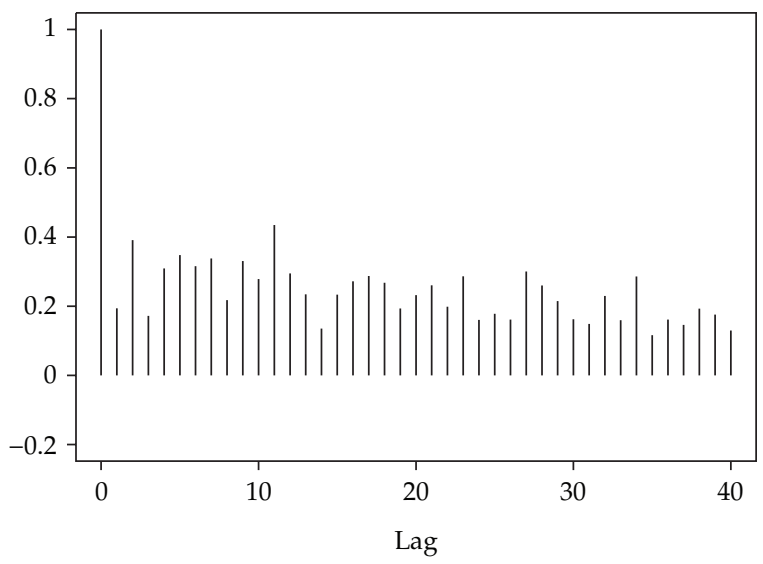

Figure 2: ACF of return of S\&P500 (square transformed).

is well defined. It is seen that, if the model (2.7) is valid, we have to assume that $\left\{\boldsymbol{\epsilon}_{t}\right\}$ is also long-memory dependent and is correlated with $\left\{\mathbf{Z}_{t}\right\}$.

Hence, we suppose that $\left\{\mathbf{Z}_{t}\right\}$ and $\left\{\boldsymbol{\varepsilon}_{t}\right\}$ are defined by

$$
\begin{aligned}
& \mathbf{Z}_{t}=\sum_{j=0}^{\infty} \boldsymbol{r}_{j} \mathbf{a}_{t-j}+\sum_{j=0}^{\infty} \boldsymbol{\rho}_{j} \mathbf{b}_{t-j}, \\
& \boldsymbol{\epsilon}_{t}=\sum_{j=0}^{\infty} \boldsymbol{\eta}_{j} \mathbf{e}_{t-j}+\sum_{j=0}^{\infty} \boldsymbol{\xi}_{j} \mathbf{b}_{t-j},
\end{aligned}
$$

where $\left\{\mathbf{a}_{t}\right\},\left\{\mathbf{b}_{t}\right\}$, and $\left\{\mathbf{e}_{t}\right\}$ are $p$-dimensional zero-mean uncorrelated processes, and they are mutually independent. Here the coefficients $\left\{\boldsymbol{r}_{j}\right\}$ and $\left\{\boldsymbol{\rho}_{j}\right\}$ are $p \times p$-matrices, and all the components of $\boldsymbol{r}_{j}$ are $\ell^{1}$-summable, (for short, $\boldsymbol{r}_{j} \in \ell^{1}$ ) and those of $\boldsymbol{\rho}_{j}$ are $\ell^{2}$-summable (for 
short, $\boldsymbol{\rho}_{j} \in \ell^{2}$ ). The coefficients $\left\{\boldsymbol{\eta}_{j}\right\}$ and $\left\{\boldsymbol{\xi}_{j}\right\}$ are $n \times p$-matrices, $\boldsymbol{\eta}_{j} \in \ell^{1}$, and $\boldsymbol{\xi}_{j} \in \ell^{2}$. From (2.9) it follows that

$$
\mathbf{Y}_{t}=\boldsymbol{\alpha}+\sum_{j=0}^{\infty}\left(\mathbf{B}^{\prime} \boldsymbol{\gamma}_{j} \mathbf{a}_{t-j}+\boldsymbol{\eta}_{j} \mathbf{e}_{t-j}\right)+\sum_{j=0}^{\infty}\left(\mathbf{B}^{\prime} \boldsymbol{\rho}_{j}+\boldsymbol{\xi}_{i}\right) \mathbf{b}_{t-j}
$$

Although $\left(\mathbf{B}^{\prime} \boldsymbol{\rho}_{j}+\boldsymbol{\xi}_{j}\right) \in \ell^{2}$ generally, if $\mathbf{B}^{\prime} \boldsymbol{\rho}_{j}+\boldsymbol{\xi}_{j}=O\left(1 / j^{\alpha}\right), \alpha>1$, then $\left(\mathbf{B}^{\prime} \boldsymbol{\rho}_{j}+\boldsymbol{\xi}_{j}\right) \in \ell^{1}$, which leads to the following.

Proposition 2.1. If $\mathbf{B}^{\prime} \boldsymbol{\rho}_{j}+\boldsymbol{\xi}_{j}=O\left(j^{-\alpha}\right), \alpha>1$, then the process $\left\{\mathbf{Y}_{t}\right\}$ is short-memory dependent.

Proposition 2.1 provides an important view for the CAPM; that is, if we assume natural conditions on (2.7) based on the empirical studies, then they impose a sort of "curved structure": $\mathbf{B}^{\prime} \boldsymbol{\rho}_{j}+\boldsymbol{\xi}_{j}=O\left(j^{-\alpha}\right)$ on the regressor and disturbance. More important view is the statement implying that the process $\left\{\boldsymbol{\beta}_{i}^{\prime} \mathbf{Z}_{t}+\epsilon_{i, t}\right\}$ is fractionally cointegrated. Here $\boldsymbol{\beta}_{i}$ and $\epsilon_{i, t}$ are called the cointegrating vector and error, respectively, (see Robinson and Yajima [13]).

\section{Two-Stage Least Squares Estimation}

This section discusses estimation of (2.7) satisfying Proposition 2.1. Sinc $E\left(\mathbf{Z}_{t} \boldsymbol{\epsilon}_{t}^{\prime}\right) \neq 0$, the least squares estimator for $\mathbf{B}$, is known to be inconsistent. In what follows we assume that $\boldsymbol{\alpha}=\mathbf{0}$ in (2.7), because it can be estimated consistently by the sample mean. However, by use of the econometric theory, it is often possible to find other variables that are uncorrelated with the errors $\boldsymbol{\epsilon}_{t}$, which we call instrumental variables, and to overcome this difficulty. Without instrumental variables, correlations between the observables $\left\{\mathbf{Z}_{t}\right\}$ and unobservables $\left\{\boldsymbol{\epsilon}_{t}\right\}$ persistently contaminate our estimator for $\mathbf{B}$. Hence, instrumental variables are useful in allowing us to estimate $\mathbf{B}$.

Let $\left\{\mathbf{X}_{t}\right\}$ be $r \times 1$-dimensional vector $(p \leq r)$ instrumental variables with $E\left[\mathbf{X}_{t}\right]=\mathbf{0}$, $\operatorname{Cov}\left(\mathbf{X}_{t}, \mathbf{Z}_{t}\right) \neq \mathbf{0}$, and $\operatorname{Cov}\left(\mathbf{X}_{t}, \boldsymbol{\epsilon}_{t}\right)=\mathbf{0}$. Consider the OLS regression of $\mathbf{Z}_{t}$ on $\mathbf{X}_{t}$. If $\mathbf{Z}_{t}$ can be represented as

$$
\mathbf{Z}_{t}=\boldsymbol{\delta}^{\prime} \mathbf{X}_{t}+\mathbf{u}_{t}
$$

where $\delta$ is a $r \times p$ matrix and $\left\{\mathbf{u}_{t}\right\}$ is a $p$-dimensional vector process which is independent of $\left\{\boldsymbol{X}_{t}\right\}, \boldsymbol{\delta}$ can be estimated by the OLS estimator

$$
\widehat{\boldsymbol{\delta}}=\left[\sum_{t=1}^{T} \mathbf{X}_{t} \mathbf{X}_{t}^{\prime}\right]^{-1}\left[\sum_{t=1}^{T} \mathbf{X}_{t} \mathbf{Z}_{t}^{\prime}\right]
$$

From (2.7) with $\boldsymbol{\alpha}=\mathbf{0}$ and (3.1), $\mathbf{Y}_{t}$ has the form:

$$
\mathbf{Y}_{t}=\mathbf{B}^{\prime} \boldsymbol{\delta}^{\prime} \mathbf{X}_{t}+\mathbf{B}^{\prime} \mathbf{u}_{t}+\boldsymbol{\epsilon}_{t}
$$


and $\boldsymbol{\delta}^{\prime} \mathbf{X}_{t}$ is uncorrelated with $\mathbf{B}^{\prime} \mathbf{u}_{t}+\boldsymbol{\epsilon}_{t}$; hence, $\mathbf{B}$ can be estimated by the OLS estimator:

$$
\widehat{\mathbf{B}}_{\mathrm{OLS}}=\left[\sum_{t=1}^{T}\left(\boldsymbol{\delta}^{\prime} \mathbf{X}_{t}\right)\left(\boldsymbol{\delta}^{\prime} \mathbf{X}_{t}\right)^{\prime}\right]^{-1}\left[\sum_{t=1}^{T}\left(\boldsymbol{\delta}^{\prime} \mathbf{X}_{t}\right) \mathbf{Y}_{t}^{\prime}\right] .
$$

Using (3.2) and (3.4), we can propose the 2SLS estimator:

$$
\widehat{\mathbf{B}}_{2 \mathrm{SLS}}=\left[\sum_{t=1}^{T}\left(\widehat{\boldsymbol{\delta}}^{\prime} \mathbf{X}_{t}\right)\left(\widehat{\boldsymbol{\delta}}^{\prime} \mathbf{X}_{t}\right)^{\prime}\right]^{-1}\left[\sum_{t=1}^{T}\left(\widehat{\boldsymbol{\delta}}^{\prime} \mathbf{X}_{t}\right) \mathbf{Y}_{t}^{\prime}\right] .
$$

Now, we aim at proving the consistency and asymptotic normality of the 2SLS estima-

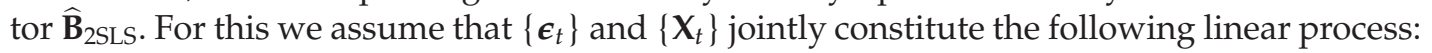

$$
\left(\begin{array}{l}
\boldsymbol{\epsilon}_{t} \\
\mathbf{X}_{t}
\end{array}\right)=\sum_{j=0}^{\infty} \mathbf{G}(j) \Gamma(t-j)=\mathbf{A}_{t}(\text { say }),
$$

where $\{\boldsymbol{\Gamma}(t)\}$ is uncorrelated $(n+r)$-dimensional vector process with

$$
\begin{gathered}
E[\boldsymbol{\Gamma}(t)]=\mathbf{0}, \\
E\left[\boldsymbol{\Gamma}(t) \boldsymbol{\Gamma}(s)^{*}\right]=\delta(t, s) \mathbf{K}, \\
\delta(t, s)= \begin{cases}1, & t=s, \\
0, & t \neq s,\end{cases}
\end{gathered}
$$

and $\mathbf{G}(j)^{\prime}$ s are $(n+r) \times(n+r)$ matrices which satisfy $\sum_{j=0}^{\infty} \operatorname{tr}\left\{\mathbf{G}(j) \mathbf{K G}(j)^{*}\right\}<\infty$. Then $\left\{\mathbf{A}_{t}\right\}$ has the spectral density matrix:

$$
\mathbf{f}(\omega)=\frac{1}{2 \pi} \mathbf{k}(\omega) \mathbf{K k}(\omega)^{*}=\left\{f_{a b}(\omega) ; 1 \leq a, b \leq(n+r)\right\} \quad(-\pi<\omega \leq \pi),
$$

where

$$
\mathbf{k}(\omega)=\sum_{j=0}^{\infty} \mathrm{G}(j) e^{i \omega j}=\left\{k_{a b}(\omega) ; 1 \leq a, b \leq(n+r)\right\} \quad(-\pi<\omega \leq \pi)
$$

Further, we assume that $\int_{-\pi}^{\pi} \log \operatorname{det} \mathbf{f}(\omega) d \omega>-\infty$, so that the process $\left\{\mathbf{A}_{t}\right\}$ is nondeterministic. For the asymptotics of $\widehat{\mathbf{B}}_{2 S L S}$, from page 108, line $\uparrow 1-$ page 109, line 7 of Hosoya [14], we impose the following assumption.

Assumption 3.1. (i) There exists $\epsilon>0$ such that, for any $t<t_{1} \leq t_{2} \leq t_{3} \leq t_{4}$ and for each $\beta_{1}, \beta_{2}$,

$$
\operatorname{var}\left[E\left\{\Gamma_{\beta_{1}}\left(t_{1}\right) \Gamma_{\beta_{2}}\left(t_{2}\right) \mid \mathbf{B}(t)\right\}-\delta\left(t_{1}-t_{2}, 0\right) K_{\beta_{1} \beta_{2}}\right]=O\left\{\left(t_{1}-t\right)^{-2-\epsilon}\right\},
$$


and also

$$
\begin{aligned}
& E\left|E\left\{\Gamma_{\beta_{1}}\left(t_{1}\right) \Gamma_{\beta_{2}}\left(t_{2}\right) \Gamma_{\beta_{3}}\left(t_{3}\right) \Gamma_{\beta_{4}}\left(t_{4}\right) \mid \mathbf{B}(t)\right\}-E\left\{\Gamma_{\beta_{1}}\left(t_{1}\right) \Gamma_{\beta_{2}}\left(t_{2}\right) \Gamma_{\beta_{3}}\left(t_{3}\right) \Gamma_{\beta_{4}}\left(t_{4}\right)\right\}\right| \\
& \quad=O\left\{\left(t_{1}-t\right)^{-1-\epsilon}\right\}
\end{aligned}
$$

uniformly in $t$, where $\mathbf{B}(t)$ is the $\sigma$-field generated by $\{\Gamma(s) ; s \leq t\}$.

(ii) For any $\epsilon>0$ and for any integer $M \geq 0$, there exists $B_{\epsilon}>0$ such that

$$
E\left[T(n, s)^{2}\left\{T(n, s)>B_{\epsilon}\right\}\right]<\epsilon,
$$

uniformly in $n, s$, where

$$
T(n, s)=\left[\sum_{\alpha, \beta=1}^{p} \sum_{r=0}^{M}\left\{\frac{1}{\sqrt{T}} \sum_{t=1}^{T}\left(\Gamma_{\alpha}(t+s) \Gamma_{\beta}(t+s+r)-K_{\alpha \beta} \delta(0, r)\right)\right\}^{2}\right]^{1 / 2},
$$

and $\left\{T(n, s)>B_{\epsilon}\right\}$ is the indicator, which is equal to 1 if $T(n, s)>B_{\epsilon}$ and equal to 0 otherwise.

(iii) Each $f_{a b}(\omega)$ is square-integrable.

Under the above assumptions, we can establish the following theorem.

Theorem 3.2. Under Assumption 3.1, it holds that

(i)

$$
\widehat{\mathbf{B}}_{2 \mathrm{SLS}} \stackrel{P}{\longrightarrow} \mathbf{B},
$$

(ii)

$$
\sqrt{T}\left(\widehat{\mathbf{B}}_{2 \mathrm{SLS}}-\mathbf{B}\right) \stackrel{d}{\longrightarrow} \mathbf{Q}^{-1} E\left[\mathbf{Z}_{t} \mathbf{X}_{t}^{\prime}\right] E\left[\mathbf{X}_{t} \mathbf{X}_{t}^{\prime}\right]^{-1} \mathbf{U}
$$

where

$$
\mathbf{Q}=\left[E\left(\mathbf{Z}_{t} \mathbf{X}_{t}^{\prime}\right)\right]\left[E\left(\mathbf{X}_{t} \mathbf{X}_{t}^{\prime}\right)\right]^{-1}\left[E\left(\mathbf{X}_{\mathbf{t}} \mathbf{Z}_{t}^{\prime}\right)\right]
$$

and $\mathbf{U}=\left\{U_{i, j} ; 1 \leq i \leq r, 1 \leq j \leq n\right\}$ is a random matrix whose elements follow normal distributions with mean 0 and

$$
\begin{aligned}
& \operatorname{Cov}\left[U_{i, j}, U_{k, l}\right]= 2 \pi \int_{-\pi}^{\pi}\left[f_{n+i, n+k}(\omega) \bar{f}_{j, l}(\omega)+f_{n+i, l}(\omega) \bar{f}_{j, n+k}(\omega)\right] d \omega \\
&+2 \pi \sum_{\beta_{1}, \ldots, \beta_{4}=1}^{p} \int_{-\pi}^{\pi} \int_{-\pi}^{\pi} \kappa_{n+i, \beta_{1}}\left(\omega_{1}\right) \kappa_{j, \beta_{2}}\left(-\omega_{1}\right) \kappa_{n+k, \beta_{3}}\left(\omega_{2}\right) \kappa_{l, \beta_{4}}\left(-\omega_{2}\right) Q_{\beta_{1}, \ldots, \beta_{4}}^{\Gamma} \\
& \times\left(\omega_{1},-\omega_{2}, \omega_{2}\right) d \omega_{1} d \omega_{2} .
\end{aligned}
$$


The next example prepares the asymptotic variance formula of $\widehat{\mathbf{B}}_{2 S L S}$ to investigate its features in simulation study.

Example 3.3. Let $\left\{Z_{t}\right\}$ and $\left\{X_{t}\right\}$ be scalar long-memory processes, with spectral densities $\left\{2 \pi\left|1-e^{i \lambda}\right|^{2 d_{z}}\right\}^{-1}$ and $\left\{2 \pi\left|1-e^{i \lambda}\right|^{2 d_{X}}\right\}^{-1}$, respectively, and cross spectral density $(1 / 2 \pi)(1-$ $\left.e^{i \lambda}\right)^{-d_{X}}\left(1-e^{-i \lambda}\right)^{-d_{Z}}$, where $0<d_{Z}<1 / 2$ and $0<d_{X}<1 / 2$. Then

$$
E\left(X_{t} Z_{t}\right)=\frac{1}{2 \pi} \int_{-\pi}^{\pi} \frac{1}{\left(1-e^{i \lambda}\right)^{d_{X}}} \frac{1}{\left(1-e^{-i \lambda}\right)^{d_{z}}} d \lambda
$$

Suppose that $\left\{\epsilon_{t}\right\}$ is a scalar uncorrelated process with $\sigma_{\epsilon}^{2} \equiv E\left\{\epsilon_{t}^{2}\right\}$. Assuming Gaussianity of $\left\{A_{t}\right\}$, it is seen that the right hand of (3.17) is

$$
2 \pi \int_{-\pi}^{\pi} \frac{1}{2 \pi\left|1-e^{i \lambda}\right|^{2 d_{X}}} \frac{\sigma_{\epsilon}^{2}}{2 \pi} d \lambda,
$$

which entails

$$
\begin{aligned}
\lim _{T \rightarrow \infty} \operatorname{var}\left[\sqrt{T}\left(\widehat{B}_{2 \text { SLS }}-B\right)\right] & =\frac{2 \pi \int_{-\pi}^{\pi}\left(1 / 2 \pi\left|1-e^{i \lambda}\right|^{2 d_{X}}\right)\left(\sigma_{\epsilon}^{2} / 2 \pi\right) d \lambda}{\left(1 / 2 \pi \int_{-\pi}^{\pi}\left(1 /\left(1-e^{i \lambda}\right)^{d_{X}}\right)\left(1 /\left(1-e^{-i \lambda}\right)^{d_{Z}}\right) d \lambda\right)^{2}} \\
& =\sigma_{\epsilon}^{2}\left(2 \pi \frac{\int_{-\pi}^{\pi}\left(1 /\left|1-e^{i \lambda}\right|^{2 d_{X}}\right) d \lambda}{\left(\int_{-\pi}^{\pi}\left(1 /\left(1-e^{i \lambda}\right)^{d_{X}}\right)\left(1 /\left(1-e^{-i \lambda}\right)^{d_{Z}}\right) d \lambda\right)^{2}}\right) \\
& =\sigma_{\epsilon}^{2} \times V_{*}\left(d_{X}, d_{Z}\right) .
\end{aligned}
$$

\section{Numerical Studies}

In this section, we evaluate the behaviour of $\widehat{\mathbf{B}}_{2 \text { SLS }}$ in the case $p=1$ in (2.7) numerically.

Example 4.1. Under the condition of Example 3.3, we investigate the asymptotic variance behaviour of $\widehat{\mathbf{B}}_{2 \text { SLS }}$ by simulation. Figure 3 plots $V_{*}\left(d_{X}, d_{Z}\right)$ for $0<d_{X}<1 / 2$ and $0<d_{Z}<1 / 2$.

From Figure 3, we observe that, if $d_{Z} \searrow 0$ and if $d_{X} \nearrow 1 / 2$, then $V_{*}$ becomes large, and otherwise $V_{*}$ is small. This result implies only in the case that the long-memory behavior of $Z_{t}$ is weak and the long-memory behavior of $X_{t}$ is strong, $V_{*}$ is large. Note that long-memory behaviour of $Z_{t}$ makes the asymptotic variance of the 2SLS estimator small, but one of $X_{t}$ makes it large. 


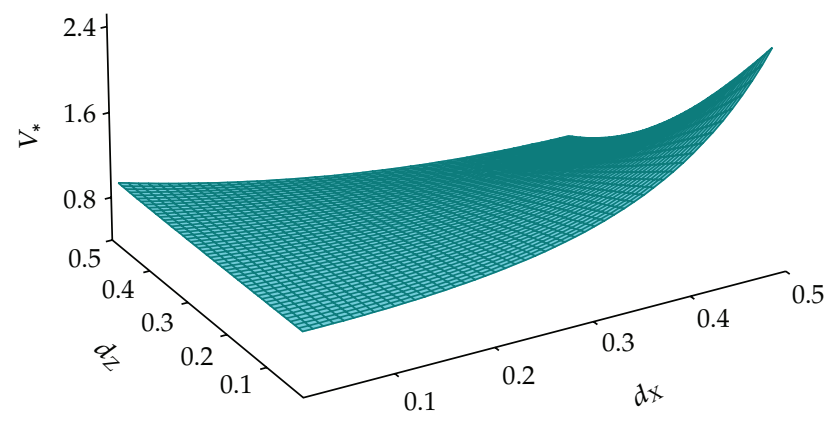

Figure 3: $V_{*}\left(d_{x}, d_{z}\right)$ in Section 4.

Table 1: MSE of $\widehat{B}_{2 S L S}$ and $\widetilde{B}_{\text {OLS }}$.

\begin{tabular}{lccc}
\hline$d_{2}$ & 0.1 & 0.2 & 0.3 \\
\hline$\widehat{B}_{2 \text { SLS }}\left(d_{1}=0.1\right)$ & 0.03 & 0.052 & 0.189 \\
$\widetilde{B}_{\text {OLS }}\left(d_{1}=0.1\right)$ & 0.259 & 0.271 & 0.34 \\
$\widehat{B}_{2 \text { SLS }}\left(d_{1}=0.2\right)$ & 0.03 & 0.075 & 0.342 \\
$\widetilde{B}_{\text {OLS }}\left(d_{1}=0.2\right)$ & 0.178 & 0.193 & 0.307 \\
$\widehat{B}_{2 \text { SLS }}\left(d_{1}=0.3\right)$ & 0.019 & 0.052 & 0.267 \\
$\widetilde{B}_{\text {OLS }}\left(d_{1}=0.3\right)$ & 0.069 & 0.089 & 0.23 \\
\hline
\end{tabular}

Example 4.2. In this example, we consider the following model:

$$
\begin{aligned}
& Y_{t}=Z_{t}+\epsilon_{t}, \\
& Z_{t}=X_{t}+u_{t}, \\
& \epsilon_{t}=w_{t}+u_{t},
\end{aligned}
$$

where $X_{t}, w_{t}$, and $u_{t}$ are the scalar long-memory processes which follow FARIMA $\left(0, d_{1}, 0\right)$, $\operatorname{FARIMA}\left(0, d_{2}, 0\right)$, and FARIMA $(0,0.1,0)$, respectively. Note that $Z_{t}$ and $\epsilon_{t}$ are correlated, $X_{t}$ and $Z_{t}$ are correlated, but $X_{t}$ and $\epsilon_{t}$ are independent. Under this model we compare $\widehat{B}_{2 S L S}$ with the ordinary least squares estimator $\widetilde{B}_{\mathrm{OLS}}$ for $B$, which is defined as

$$
\widetilde{B}_{\mathrm{OLS}}=\left[\sum_{t=1}^{T} Z_{t}^{2}\right]^{-1}\left[\sum_{t=1}^{T} Z_{t} Y_{t}\right] .
$$

The lengths of $X_{t}, Y_{t}$, and $Z_{t}$ are set by 100 , and based on 5000 times simulation we report the mean square errors (MSE) of $\widehat{B}_{2 \text { SLS }}$ and $\widetilde{B}_{\text {OLS }}$. We set $d_{1}, d_{2}=0.1,0.2,0.3$ in Table 1 .

In most cases of $d_{1}$ and $d_{2}$ in Table 1 , MSE of $\widehat{B}_{2 S L S}$ is smaller than that of $\widetilde{B}_{\text {OLS. }}$. Hence, from this Example we can see that our estimator $\widehat{B}_{2 S L S}$ is better than $\widetilde{B}_{\text {OLS }}$ in the sense of MSE. Furthermore, from Table 1, we can see that MSE of $\widehat{B}_{2 S L S}$ and $\widetilde{B}_{\text {OLS }}$ increases as $d_{2}$ becomes large; that is, long-memory behavior of $w_{t}$ makes the asymptotic variances of $\widehat{B}_{2 \text { SLS }}$ and $\widetilde{B}_{\text {OLS }}$ large. 
Table 2: $\widehat{B}_{2 S L S}$ based on the actual financial data.

\begin{tabular}{lccccc}
\hline Stock & IBM & Nike & Amazon & American Express & Ford \\
\hline$\widehat{\mathbf{B}}_{\text {2SLS }}$ & 0.75 & 1.39 & 1.71 & 2.61 & -1.89 \\
\hline
\end{tabular}

Example 4.3. In this example, we calculate $\widehat{\mathbf{B}}_{2 S L S}$ based on the actual financial data. We choose S\&P500 (square transformed) as $\mathbf{Z}_{t}$ and the Nikkei stock average as an instrumental variable $\mathbf{X}_{t}$. Assuming that $\mathbf{Y}_{t}(5 \times 1)$ consists of the return of IBM, Nike, Amazon, American Expresses and Ford; the 2SLS estimates for $B_{i}, i=1, \ldots, 5$ are recorded in Table 2 . We chose the Nikkei stock average as the instrumental variable, because we got the following correlation analysis between the residual processes of returns and Nikkei.

Correlation of IBM's residual and Nikkei's return: -0.000311

Correlation of Nike's residual and Nikkei's return: -0.00015

Correlation of Amazon's residual and Nikkei's return: -0.000622

Correlation of American Express's residual and Nikkei's return: 0.000147

Correlation of Ford's residual and Nikkei's return: -0.000536 ,

which supports the assumption $\operatorname{Cov}\left(\mathbf{X}_{t}, \boldsymbol{\varepsilon}_{t}\right)=\mathbf{0}$.

From Table 2, we observe that the return of the finance stock (American Express) is strongly correlated with that of S\&P500 and the return of the auto industry stock (Ford) is negatively correlated with that of S\&P500.

\section{Proof of Theorem}

This section provides the proof of Theorem 3.2. First for convenience we define $\widehat{\mathbf{Z}}_{t}=$ $\left(\widehat{Z}_{1, t}, \ldots, \widehat{Z}_{p, t}\right)^{\prime} \equiv \widehat{\boldsymbol{\delta}}^{\prime} \mathbf{X}_{t}$. Let $\widehat{\mathbf{u}}_{t}=\left(\widehat{u}_{1, t}, \ldots, \widehat{u}_{p, t}\right)^{\prime}$ be the residual from the OLS estimation of (3.1); that is,

$$
\widehat{u}_{i, t}=Z_{i, t}-\widehat{Z}_{i, t}
$$

The OLS makes this residual orthogonal to $\mathbf{X}_{t}$ :

$$
\sum_{t=1}^{T} \mathbf{X}_{t}^{\prime} \widehat{u}_{i, t}=\mathbf{0}
$$

which implies the residual is orthogonal to $\widehat{Z}_{j, t}$,

$$
\sum_{t=1}^{T} \widehat{Z}_{j, t} \widehat{u}_{i, t}=\left(\sum_{t=1}^{T} \mathbf{X}_{t}^{\prime} \widehat{u}_{i, t}\right) \widehat{\boldsymbol{\delta}}_{j}=0,
$$


where $\widehat{\boldsymbol{\delta}}_{j}$ is $j$ th column vector of $\widehat{\boldsymbol{\delta}}$. Hence, we can obtain

$$
\sum_{t=1}^{T} \widehat{Z}_{j, t} Z_{i, t}=\sum_{t=1}^{T} \widehat{Z}_{j, t}\left(\widehat{Z}_{i, t}+\widehat{u}_{i, t}\right)=\sum_{t=1}^{T} \widehat{Z}_{j, t} \widehat{Z}_{i, t}
$$

for all $i$ and $j$. This means

$$
\sum_{t=1}^{T} \widehat{\mathbf{Z}}_{t} \mathbf{Z}_{t}^{\prime}=\sum_{t=1}^{T} \widehat{\mathbf{Z}}_{t} \widehat{\mathbf{Z}}_{t}^{\prime}
$$

So, the $i$ th column vector of the 2SLS estimator (3.5) $\widehat{\boldsymbol{\beta}}_{2 \text { SLS }, i}$ (say) can be represented as

$$
\widehat{\boldsymbol{\beta}}_{2 \mathrm{SLS}, i}=\left[\sum_{t=1}^{T} \widehat{\mathbf{Z}}_{t} \mathbf{Z}_{t}^{\prime}\right]^{-1}\left[\sum_{t=1}^{T} \widehat{\mathbf{Z}}_{t} Y_{i, t}\right],
$$

which leads to

$$
\widehat{\boldsymbol{\beta}}_{2 \mathrm{SLS}, i}-\boldsymbol{\beta}_{i}=\left[\frac{1}{T} \sum_{t=1}^{T} \widehat{\mathbf{Z}}_{t} \mathbf{Z}_{t}^{\prime}\right]^{-1}\left[\frac{1}{T} \sum_{t=1}^{T} \widehat{\mathbf{Z}}_{t} \epsilon_{i, t}\right] .
$$

Hence, we can see that

$$
\sqrt{T}\left(\widehat{\mathbf{B}}_{2 \mathrm{SLS}}-\mathbf{B}\right)=\left[\frac{1}{T} \sum_{t=1}^{T} \widehat{\mathbf{Z}}_{t} \mathbf{Z}_{t}^{\prime}\right]^{-1}\left[\frac{1}{\sqrt{T}} \sum_{t=1}^{T} \widehat{\mathbf{Z}}_{t} \boldsymbol{\epsilon}_{t}^{\prime}\right] .
$$

Note that, by the ergodic theorem (e.g., Stout [15] p179-181),

$$
\begin{aligned}
\frac{1}{T} \sum_{t=1}^{T} \widehat{\mathbf{Z}}_{t} \mathbf{Z}_{t}^{\prime} & =\frac{1}{T} \widehat{\boldsymbol{\delta}}^{\prime} \sum_{t=1}^{T} \mathbf{X}_{t} \mathbf{Z}_{t}^{\prime} \\
& =\left[\frac{1}{T} \sum_{t=1}^{T} \mathbf{Z}_{t} \mathbf{X}_{t}^{\prime}\right]\left[\frac{1}{T} \sum_{t=1}^{T} \mathbf{X}_{t} \mathbf{X}_{t}^{\prime}\right]^{-1}\left[\frac{1}{T} \sum_{t=1}^{T} \mathbf{X}_{t} \mathbf{Z}_{t}^{\prime}\right] \\
& \stackrel{P}{\longrightarrow} \mathbf{Q} .
\end{aligned}
$$

Furthermore, the second term of the right side of (5.8) can be represented as

$$
\left[\frac{1}{\sqrt{T}} \sum_{t=1}^{T} \widehat{\mathbf{Z}}_{t} \boldsymbol{\epsilon}_{t}^{\prime}\right]=\widehat{\boldsymbol{\delta}}^{\prime} \frac{1}{\sqrt{T}} \sum_{t=1}^{T} \mathbf{X}_{t} \boldsymbol{\epsilon}_{t}^{\prime}
$$


and by the ergodic theorem (e.g., Stout [15] p179-181), we can see

$$
\widehat{\boldsymbol{\delta}}^{\prime}=\left[\sum_{t=1}^{T} \mathbf{Z}_{t} \mathbf{X}_{t}^{\prime}\right]\left[\sum_{t=1}^{T} \mathbf{X}_{t} \mathbf{X}_{t}^{\prime}\right]^{-1} \stackrel{P}{\longrightarrow}\left[E\left(\mathbf{Z}_{t} \mathbf{X}_{t}^{\prime}\right)\right]\left[E\left(\mathbf{X}_{t} \mathbf{X}_{t}^{\prime}\right)\right]^{-1}
$$

Proof of (i). From the above,

$$
\widehat{\mathbf{B}}_{2 \mathrm{SLS}}-\mathbf{B}=O_{P}\left[\frac{1}{T} \sum_{t=1}^{T} \mathbf{X}_{t} \boldsymbol{\epsilon}_{t}^{\prime}\right]
$$

In view of Theorem 1.2 (i) of Hosoya [14], the right-hand side of (5.12) converges to 0 in probability.

Proof of (ii). From Theorem 3.2 of Hosoya [14], if Assumption 3.1 holds, it follows that $(1 / \sqrt{T}) \sum_{t=1}^{T} \mathbf{X}_{t} \boldsymbol{\epsilon}_{t}^{\prime} \stackrel{d}{\rightarrow} \mathbf{U}$. Hence, Theorem 3.2 is proved.

\section{Acknowledgments}

The author would like to thank the Editor and the referees for their comments, which improved the original version of this paper.

\section{References}

[1] H. Markowitz, Portfolio Selection: Efficient Diversification of Investments, John Wiley \& Sons, New York, NY, USA, 1991.

[2] W. Sharpe, "Capital asset prices: a theory of market equilibrium under conditions of risk," Journal of Finance, vol. 19, pp. 425-442, 1964.

[3] J. Lintner, "The valuation of risk assets and the selection of risky in vestments in stock portfolios and capital budgets," The Review of Economics and Statistics, vol. 47, pp. 13-37, 1965.

[4] F. Black, "Capital market equilibrium with restricted borrowing," The Journal of Business, vol. 45, pp. $444-455,1972$.

[5] J. Y. Campbell, A. W. Lo, and A. C. Mackinlay, The Econometrics of Financial Markets, Princeton University Press, Princeton, NJ, USA, 1997.

[6] C. W. J. Granger, "Long memory relationships and the aggregation of dynamic models," Journal of Econometrics, vol. 14, no. 2, pp. 227-238, 1980.

[7] P. G. Wright, The Tariff on Animal and Vegetable Oils, Macmillan, New York, NY, USA, 1928.

[8] O. Reiersøl, "Confluence analysis by means of instrumental sets of variables," Arkiv för Matematik, Astronomi Och Fysik, vol. 32A, no. 4, pp. 1-119, 1945.

[9] R. C. Geary, "Determination of linear relations between systematic parts of variables with errors of observation the variances of which are unknown," Econometrica, vol. 17, pp. 30-58, 1949.

[10] H. White, Asymptotic Theory for Econometricians, Academic Press, New York, NY, USA, 2001.

[11] P. M. Robinson and F. J. Hidalgo, "Time series regression with long-range dependence," The Annals of Statistics, vol. 25, no. 1, pp. 77-104, 1997.

[12] K. Choy and M. Taniguchi, "Stochastic regression model with dependent disturbances," Journal of Time Series Analysis, vol. 22, no. 2, pp. 175-196, 2001.

[13] P. M. Robinson and Y. Yajima, "Determination of cointegrating rank in fractional systems," Journal of Econometrics, vol. 106, no. 2, pp. 217-241, 2002.

[14] Y. Hosoya, "A limit theory for long-range dependence and statistical inference on related models," The Annals of Statistics, vol. 25, no. 1, pp. 105-137, 1997.

[15] W. F. Stout, Almost Sure Convergence, Academic Press, New York, NY, USA, 1974. 


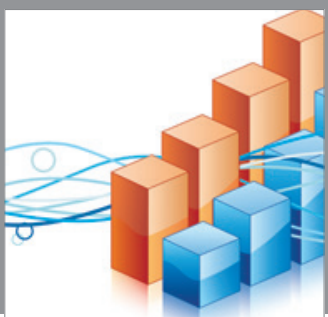

Advances in

Operations Research

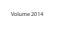

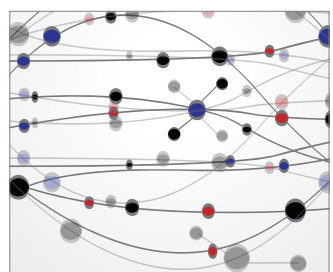

\section{The Scientific} World Journal
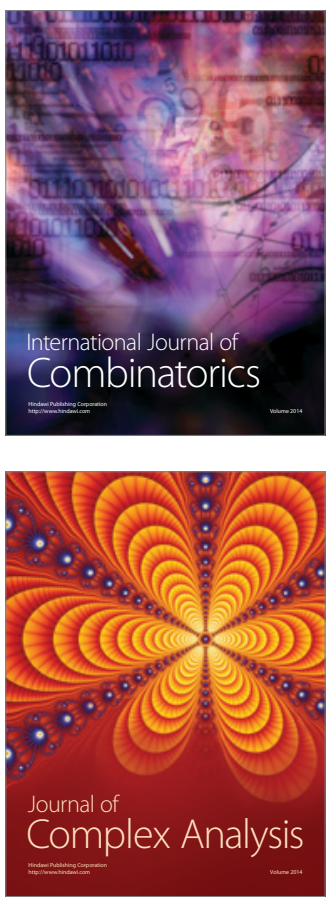

International Journal of

Mathematics and

Mathematical

Sciences
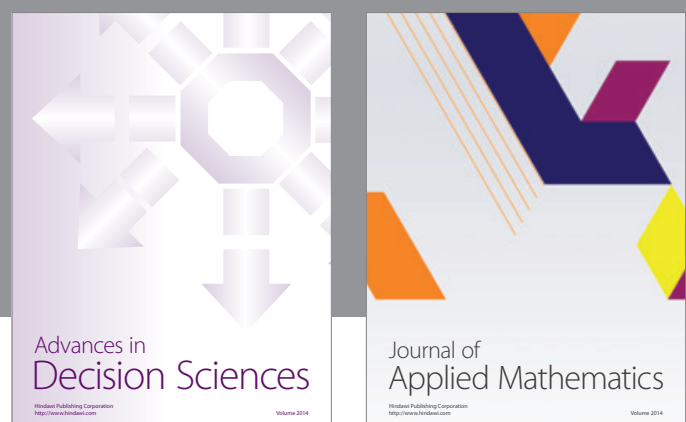

Journal of

Applied Mathematics
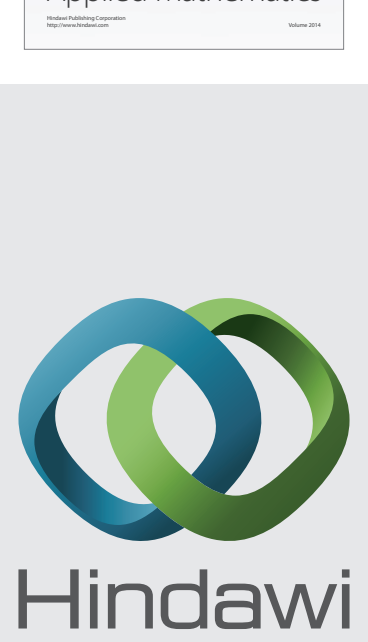

Submit your manuscripts at http://www.hindawi.com
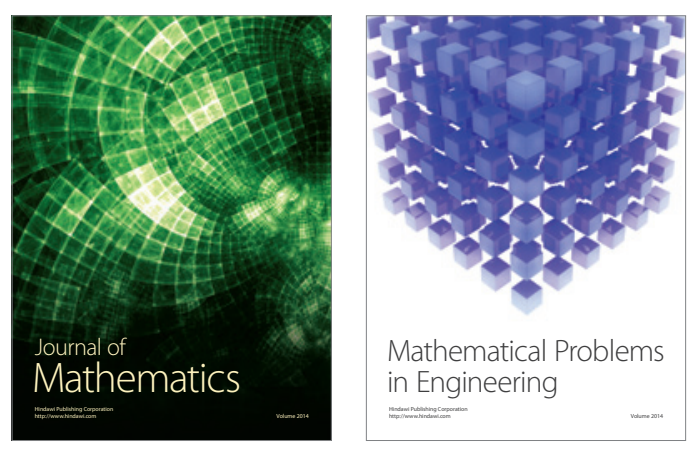

Mathematical Problems in Engineering
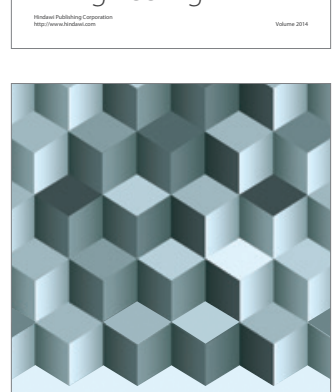

Journal of

Function Spaces
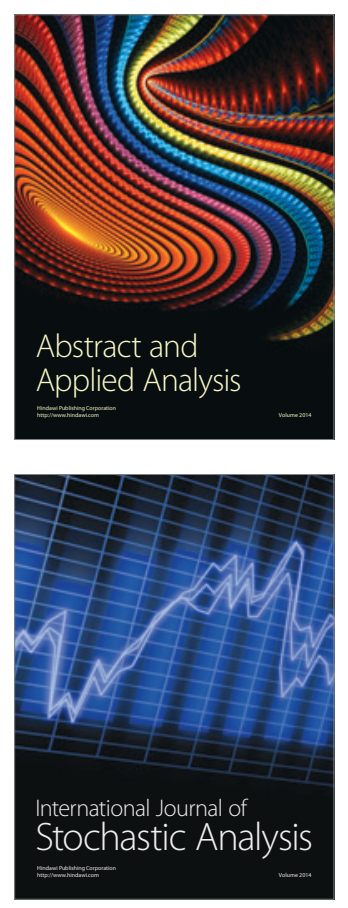

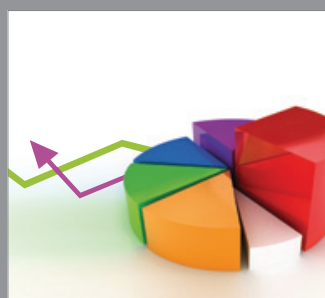

ournal of

Probability and Statistics

Promensencen
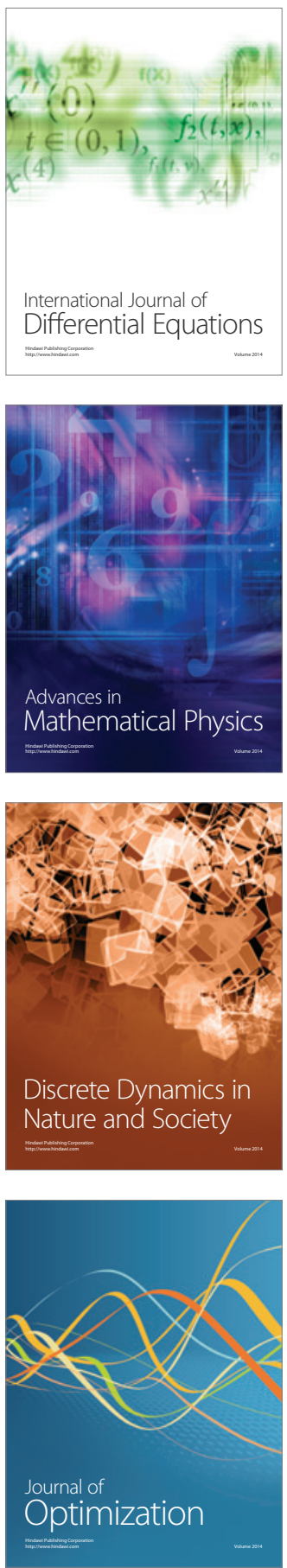